\title{
A bumpy, holey method to probe proteins
}

A 'bump-and-hole' strategy probes individual BET bromodomains.

Bromo and extraterminal (BET) proteins play significant roles in epigenetics, transcriptional regulation and cancer and are therefore important drug targets. BET proteins each contain two bromodomains that bind to acetylated lysines in histone tails, directing recruitment to nucleosomes. But like protein kinases, another important class of drug targets, BET bromodomain binding sites are highly homologous, making it a challenge to specifically inhibit individual family members or to dissect their individual functions.

More than a decade ago, Kevan Shokat's laboratory (now at University of California, San Francisco) developed a clever approach to target individual protein kinases, using a bump-and-hole strategy. To selectively engineer a kinase, they mutated a larger amino acid at the ATP-binding pocket to a smaller one, creating a tiny 'hole' that would accept an inhibitor containing an additional 'bump'. Wild-type kinases would not bind the bumpy inhibitor owing to steric hindrance.

Alessio Ciulli of the University of Dundee and University of Cambridge, and colleagues, have now ported this strategy to BET bromodomains. They inspected crystal structures of these proteins in complex with inhibitors and identified a leucine residue at the binding site, which they then mutated to a smaller alanine residue to create a hole. After confirming that mutant BET proteins were reasonably stable and functional, they turned to an inhibitor currently in clinical trials called I-BET. They looked at the crystal structures again to see how this small molecule was binding in the pocket and then designed a version of I-BET containing a bump —an additional alkyl group.

They solved a new crystal structure of a mutant BET protein, Brd2, in complex with bulked-up I-BET inhibitors, showing that an additional ethyl group on the inhibitor filled the holey binding pocket well. Then they tested the selectivity and affinity of bumpy I-BET across the entire BET bromodomain subfamily, showing that the bulkier ligand was specific for binding target holey mutant family members. Last, they tested the performance of the engineered system in cells, showing that bumpy I-BET was able to displace mutant Brd4 from chromatin.

Besides providing a useful method for functionally profiling an interesting class of proteins, this work also proves the general power of the bump-and-hole approach not only for small-molecule binding interactions but also for protein-protein interactions.

\section{Allison Doerr}

\section{RESEARCH PAPERS}

Baud, M.G.J. et al. A bump-and-hole approach to engineer controlled selectivity of BET bromodomain chemical probes. Science 346, 638-641 (2014). 